\title{
Recurrent Disease, Primary Site
}

National Cancer Institute

\section{Source}

National Cancer Institute. Recurrent Disease, Primary Site. NCI Thesaurus. Code C55072.

Recurrence of symptoms of a disease at the original site. 